\title{
Genetic Parameters and Combining Ability Effects of Parents for Seed Yield and other Quantitative Traits in Black Gram [Vigna mungo (L.) Hepper]
}

\author{
Supriyo CHAKRABORTY, Hironya K. BORAH, Birinchi K. BORAH, Dalim \\ PATHAK, Bipin K. BARUAH, Hemen KALITA, Bhubaneswar BARMAN \\ Assam Agricultural University, Regional Agricultural Research Station, Nagaon 782 002, \\ Assam,India; supriyoch_2008@rediffmail.com (corresponding author)
}

\begin{abstract}
Line $\mathrm{x}$ tester analysis was carried out in black gram [Vigna mungo (L.) Hepper], an edible legume, to estimate the gca (general combining ability) effects of parents (3 lines and 3 testers) and the SCA (specific combining ability) effects of 9 crosses for seed yield and other eleven quantitative traits. Though additive and nonadditive gene actions governed the expression of quantitative traits, the magnitude of nonadditive gene action was higher than that of additive gene action for each quantitative trait. Two parents viz. 'UG157' and 'DPU915' were good general combiners. Two crosses namely 'PDB 88-31'/'DPU 915' and 'PLU 277'/'KAU7' had high per se performance along with positive significant $S C A$ effect for seed yield/plant. The degree of dominance revealed overdominance for all the traits except clusters/plant with partial dominance. The predictability ratio also revealed the predominant role of nonadditive gene action in the genetic control of quantitative traits. Narrow sense heritability was also low for each trait. Recurrent selection or biparental mating followed by selection which can exploit both additive and nonadditive gene actions would be of interest for yield improvement in black gram. Due to presence of high magnitude of nonadditive gene action, heterosis breeding could also be attempted to develop low cost hybrid variety using genetic male sterility system in black gram.
\end{abstract}

Keywords: combining ability, line x tester analysis, quantitative traits, Vigna mungo, yield

\section{Introduction}

Black gram [Vigna mungo (L.) Hepper] is an important pulse crop of India. It is mainly cultivated as a source of dietary protein because of its high protein content which is of about 25\% in seeds (Haytowitz and Mathews, 1986). Legumes like black gram can potentially fix about $80 \%$ of its own nitrogen needs through biological nitrogen fixation and in addition can contribute to the yield of subsequent crops (Lauren et al., 1998). In Assam, black gram is grown in summer and rainy seasons under rainfed condition, which adversely affects its seed yield. Average productivity of black gram in the State of Assam is low (1 $\mathrm{t} / \mathrm{ha}$ ) which is much lower than the national average. Low productivity of black gram has led to its high market price, almost three fold of rice, which is the major cereal crop of the region.

In order to increase the productivity of black gram it is essential to develop a high yielding pureline variety by selection from the segregating generations of superior crosses involving superior parents. The development of a hybrid variety exploiting heterosis is not very feasible in Assam in view of the poor socio-economic condition of the farmers. The farmers may not be able to afford the high cost of hybrid seed every season in addition to high costs of other inputs like fertilizer, pesticides etc. required for hybrids. So the development of an improved pureline variety is more desirable than hybrid variety in black gram.
Line $\mathrm{x}$ tester analysis, as proposed by Kempthorne (1957), measures the combining ability of parents to be used in hybridization and helps the crop breeder in choosing the desirable parents for hybridization program. Besides this, line $\mathrm{x}$ tester analysis also guides the breeder in choosing appropriate breeding method by estimating the $G C A$ (general combining ability) and SCA (specific combining ability) variances governing the traits and by determining the components of genetic variance viz additive and dominance variances assuming epistasis being absent. The present study was undertaken to study the $G C A$ and $S C A$ effects and variances of some black gram parents and crosses, respectively for seed yield and other quantitative traits. Genetic parameters like narrow sense heritability and degree of dominance for seed yield and other quantitative traits were also estimated in this study.

\section{Materials and methods}

Six genetically diverse but highly adapted black gram parents (namely 'UG157', 'PLU-277', 'PDB88-31', 'PU30', 'DPU915', 'KAU7') selected on the basis of previous screening were crossed in a line $\mathrm{x}$ tester mating design in rainy season. Out of these, three genotypes viz. 'UG157', 'PLU-277' and 'PDB88-31' were used as lines and the remaining three genotypes as testers in hybridization. Nine $\mathrm{F}_{1} \mathrm{~s}$ and six parents were planted in randomized block design with 3 replications in a 4-row plot of $5 \mathrm{~m}$ length hav- 
122

ing $40 \mathrm{~cm} \times 10 \mathrm{~cm}$ crop geometry in the next rainy season at RARS, Nagaon, Assam. Data were recorded on ten randomly selected plants per replication for seed yield per plant and other eleven quantitative traits (Tab. 1).

Mean data were analyzed to obtain the $G C A$ and $S C A$ estimates following the procedure of line $\mathrm{x}$ tester analysis given by Kempthorne (1957). The relative importance of $G C A$ and $S C A$ variances was computed as predictability ratio as suggested by Baker (1978) along with the estimation of degree of dominance and narrow sense heritability for all the traits.

\section{Results and discussion}

\section{Analysis of variance}

The analysis of variance revealed that the lines differed among themselves at $\mathrm{p}=0.01$ or 0.05 for five characters namely days to $50 \%$ flowering, days to maturity, clusters/ plant, root weight/plant and harvest index (Tab. 1). The testers differed amongst themselves at $\mathrm{p}=0.01$ for seven characters viz. plant height, days to $50 \%$ flowering, days to maturity, clusters per plant, seed yield/plant and harvest index. But neither the lines nor the testers differed amongst themselves for four characters namely pods/cluster, pod length, seeds/pod and root-shoot length ratio. The proportion of mean squares due to lines was greater than that due to testers for days to $50 \%$ flowering, pod length, root-shoot length ratio, root weight/plant and harvest index. But the proportion of testers' contribution was greater than that due to lines for the remaining seven characters. Therefore, the analysis of variance suggested the presence of wide variability for the respective traits among the lines and the testers.

This justified the use of these six genotypes as parents in the present line $x$ tester analysis. Higher magnitude of the interaction component due to lines $\mathrm{x}$ testers than either due to lines or testers indicated the predominant role of nonadditive gene action (dominance) in the expression of plant height, pods/cluster, pod length and root weight/ plant. But lower magnitude of interaction component than either due to lines or testers indicated the preponderance of additive gene action in the expression of remaining eight characters, namely days to $50 \%$ flowering, days to maturity, clusters/plant, pods/plant, seeds/pod, root-shoot length ratio, seed yield/plant and harvest index (Cheralu et al., 1999). No parent showed the best per se performance for all the quantitative traits although 'DPU915' recorded the highest seed yield/plant and harvest index, followed by the parents 'PLU227' and 'KAU7'. Comparison of the per se performance for seed yield/plant among nine crosses revealed that the cross 'PDB88-31'/'DPU915' was the best cross followed by 'UG157'/'DPU915', 'PDB88-31' /'PU30' and 'PLU277'/'KAU7'. These crosses are likely to produce transgressive segregants for seed yield/plant in succeeding generations.

The interaction component due to lines $\mathrm{x}$ testers was of higher magnitude than either due to lines or testers for plant height, pods/cluster, pod length and root-weight/ plant. But the interaction component was of lower magnitude than either due to lines or testers for the remaining eight traits.

Amongst the six parents ( 3 lines and 3 testers), 'DPU915' showed the highest seed yield/plant and harvest index followed by 'PLU277' and 'KAU7' (Tab. 2). No parent showed the best per se performance for all the quantitative traits. This could be due to differential internal adjustments between seed yield/plant and its components in different parents.

A perusal of the per se performance of nine line $\mathrm{x}$ tester crosses in $F_{1}$ generation for different traits showed

Tab.1. ANOVA for line $\mathrm{x}$ tester analysis in black gram

\begin{tabular}{|c|c|c|c|c|c|c|c|c|c|c|c|c|c|}
\hline \multirow[b]{2}{*}{ Source } & \multirow[b]{2}{*}{ df } & \multicolumn{12}{|c|}{ Mean Squares } \\
\hline & & $\begin{array}{l}\text { Plant } \\
\text { height } \\
(\mathrm{cm})\end{array}$ & $\begin{array}{c}\text { Days } \\
\text { to } 50 \% \\
\text { flowering }\end{array}$ & $\begin{array}{l}\text { Days to } \\
\text { maturity }\end{array}$ & $\begin{array}{c}\text { Clusters } \\
\text { per } \\
\text { plant }\end{array}$ & $\begin{array}{l}\text { Pods } \\
\text { per } \\
\text { cluster }\end{array}$ & $\begin{array}{l}\text { Pods } \\
\text { per } \\
\text { plant }\end{array}$ & $\begin{array}{c}\text { Seeds } \\
\text { per } \\
\text { pod }\end{array}$ & $\begin{array}{l}\text { Pod } \\
\text { length } \\
(\mathrm{cm})\end{array}$ & $\begin{array}{c}\text { Root- } \\
\text { shoot } \\
\text { length } \\
\text { ratio } \\
\end{array}$ & $\begin{array}{c}\text { Root } \\
\text { Weight } \\
\text { per } \\
\text { plant }(\mathrm{g})\end{array}$ & $\begin{array}{c}\text { Seed } \\
\text { yield per } \\
\text { plant } \\
(\mathrm{g})\end{array}$ & $\begin{array}{c}\text { Harvest } \\
\text { index }\end{array}$ \\
\hline $\begin{array}{l}\text { Replica- } \\
\text { tions }\end{array}$ & 2 & 22.95 & 0.20 & 1.76 & 13.63 & 0.16 & 72.03 & 0.29 & 0.08 & 0.004 & 0.37 & 7.12 & 0.005 \\
\hline Genotypes & 14 & $498.47^{* *}$ & $32.39^{* *}$ & $19.46^{* *}$ & $160.13^{* *}$ & $1.95^{*}$ & $700.26^{* *}$ & 0.83 & $0.79^{* *}$ & $0.038^{* *}$ & $1.02^{* *}$ & $11.25^{* *}$ & $0.040^{* *}$ \\
\hline Parents & 5 & $665.66^{* *}$ & $4.19^{* *}$ & $12.22^{* *}$ & $64.26^{* *}$ & $1.92^{*}$ & $376.86^{* *}$ & 0.46 & $0.69^{* *}$ & $0.078^{* *}$ & 0.11 & 0.63 & $0.020^{* *}$ \\
\hline $\begin{array}{l}\text { Parents vs. } \\
\text { Crosses }\end{array}$ & 1 & 272.00 & $164.88^{* *}$ & $13.33^{* *}$ & $1245.93^{* *}$ & $12.89^{* *}$ & $4538.70^{* *}$ & $6.84^{* *}$ & $4.31^{* *}$ & $0.080^{* *}$ & $2.99^{* *}$ & $79.39^{* *}$ & $0.360^{* *}$ \\
\hline Crosses & 8 & $422.28^{* *}$ & $33.46^{* *}$ & $24.75^{* *}$ & $84.32^{* *}$ & 0.59 & $422.58^{* *}$ & 0.32 & $0.41^{*}$ & 0.003 & $1.34^{* *}$ & $9.36^{* *}$ & $0.020^{* *}$ \\
\hline Lines & 2 & 230.26 & $42.70^{* *}$ & $14.34^{* *}$ & $26.82^{*}$ & 0.26 & 41.34 & 0.26 & 0.47 & 0.009 & $1.35^{* *}$ & 5.71 & $0.025^{* *}$ \\
\hline Testers & 2 & $482.48^{* *}$ & $40.15^{* *}$ & $49.34^{* *}$ & $263.37^{* *}$ & 0.93 & $1098.00^{* *}$ & 0.48 & 0.11 & 0.003 & 0.74 & $16.73^{* *}$ & $0.010^{* *}$ \\
\hline $\begin{array}{l}\text { Lines } \mathrm{x} \\
\text { Testers }\end{array}$ & 4 & $488.20^{* *}$ & $25.49^{* *}$ & $17.67^{* *}$ & $23.54^{* *}$ & $2.63^{*}$ & $275.44^{*}$ & 0.26 & $0.53^{*}$ & 0.001 & $1.64^{* *}$ & $7.52^{* *}$ & $0.010^{* *}$ \\
\hline Error & 28 & 71.78 & 0.46 & 0.21 & 5.22 & 0.70 & 68.45 & 0.67 & 0.17 & 0.006 & 0.25 & 1.81 & 0.001 \\
\hline
\end{tabular}

${ }^{*},{ }^{* *}$ Significant at $5 \%$ and $1 \%$ levels, respectively 
Tab. 2. Per se performance of lines and testers for different quantitative traits in black gram

\begin{tabular}{|c|c|c|c|c|c|c|c|c|c|c|c|c|}
\hline Parent & $\begin{array}{l}\text { Plant } \\
\text { height } \\
(\mathrm{cm})\end{array}$ & $\begin{array}{c}\text { Days } \\
\text { to } 50 \% \\
\text { flowering }\end{array}$ & $\begin{array}{l}\text { Days to } \\
\text { maturity }\end{array}$ & $\begin{array}{c}\text { Clusters } \\
\text { per } \\
\text { plant }\end{array}$ & $\begin{array}{l}\text { Pods } \\
\text { per } \\
\text { cluster }\end{array}$ & $\begin{array}{l}\text { Pods } \\
\text { per } \\
\text { plant }\end{array}$ & $\begin{array}{l}\text { Seeds } \\
\text { per } \\
\text { pod }\end{array}$ & $\begin{array}{l}\text { Pod } \\
\text { length } \\
(\mathrm{cm})\end{array}$ & $\begin{array}{l}\text { Root- } \\
\text { shoot } \\
\text { length } \\
\text { ratio }\end{array}$ & $\begin{array}{c}\text { Root } \\
\text { Weight } \\
\text { per plant } \\
\text { (g) }\end{array}$ & $\begin{array}{c}\text { Seed } \\
\text { yield per } \\
\text { plant }(\mathrm{g})\end{array}$ & $\begin{array}{c}\text { Harvest } \\
\text { index }\end{array}$ \\
\hline \multicolumn{13}{|c|}{ Lines } \\
\hline 'UG157' & 54.3 & 68 & 85 & 3.3 & 2.33 & 10 & 5.3 & 3.3 & 0.32 & 1.78 & 1.69 & 0.23 \\
\hline ‘PLU277’ & 37.3 & 72 & 90 & 15.0 & 2.67 & 35 & 6.3 & 4.5 & 0.45 & 1.52 & 2.76 & 0.33 \\
\hline 'PDB88-31' & 29.3 & 70 & 85 & 11.6 & 3.00 & 26 & 5.6 & 4.0 & 0.53 & 1.91 & 2.26 & 0.31 \\
\hline \multicolumn{13}{|c|}{ Testers } \\
\hline 'PU30’ & 42.6 & 69 & 86 & 4.3 & 2.33 & 7 & 6.0 & 4.2 & 0.37 & 1.74 & 2.25 & 0.13 \\
\hline 'DPU915' & 23.3 & 71 & 88 & 8.6 & 4.33 & 27 & 6.3 & 4.7 & 0.53 & 1.57 & 2.97 & 0.36 \\
\hline 'KAU7’' & 62.6 & 70 & 86 & 5.0 & 3.67 & 13 & 6.0 & 4.0 & 0.32 & 2.02 & 2.63 & 0.32 \\
\hline General Mean & 41.6 & 70 & 87 & 8.0 & 3.06 & 19 & 5.9 & 4.1 & 0.45 & 1.75 & 2.43 & 0.28 \\
\hline
\end{tabular}

that the cross 'PDB88-31'/'DPU915' had recorded the highest seed yield/plant followed by three crosses namely 'UG157'/'DPU915', 'PDB88-31'/'PU30' and 'PLU277’/KAU7’ (Tab. 3).

\section{$G C A$ effects}

The $G C A$ effects of lines and testers are presented in (Tab. 4). The $G C A$ effect is a good estimate of additive gene action (Sprague and Tatum, 1942). Among three lines, none was the best general combiner for all the quantitative traits. But the line 'UG157' with positive $G C A$ effect for nine quantitative traits was taken as the best general combiner in this study. It is a recommended black gram variety for cultivation in Assam. Similarly, among three testers, 'DPU915' with significant positive $G C A$ effect for six traits was the best general combiner for clusters/plant, pods/plant, seed yield/plant and harvest index (positive $G C A$ values for days to $50 \%$ flowering and days to maturity were not considered as these traits will increase crop duration). The per se performance of DPU was also higher than other two testers for most of the traits.

The $G C A$ effect is a good measure of additive gene action. The study of $G C A$ effects revealed that the line
'UG157' was the best general combiner amongst lines. The tester 'DPU915' was the best general combiner amongst testers for clusters/plant, pods/plant, seed yield/plant and harvest index. So 'DPU915' could be used as one of the parents in hybridization program in future to obtain desirable recombinant for increased seed yield.

\section{$S C A$ effects}

The $S C A$ effects of the nine crosses for different quantitative traits were computed (Tab. 5). The $S C A$ effect is actually a measure of nonadditive gene action. The top ranking two crosses on the basis of per se performance, in general, exhibited positive significant $S C A$ effect for seed yield/ plant and harvest index. The cross 'PLU277'/KAU7' appeared to be the best amongst all the crosses for seed yield/ plant and harvest index as it showed positive significant $S C A$ effect and high per se performance with parents being poor general combiners. But the cross 'PDB88-31' /DPU915' showed positive significant $S C A$ effect and high per se performance for seed yield/plant. This cross had one parent ('DPU915') having good GCA effect for seed yield/plant, clusters/plant, pods/plant and harvest index.

Tab. 3. Per se performance of $9 \mathrm{~L} X \mathrm{~T}$ crosses of black gram in $\mathrm{F}_{1}$ generation for different quantitative traits

\begin{tabular}{cccccccccccccc}
\hline Cross & $\begin{array}{c}\text { Plant } \\
\text { height } \\
(\mathrm{cm})\end{array}$ & $\begin{array}{c}\text { Days } \\
\text { to 50\% } \\
\text { flowering }\end{array}$ & $\begin{array}{c}\text { Days to } \\
\text { maturity }\end{array}$ & $\begin{array}{c}\text { Clusters } \\
\text { per plant }\end{array}$ & $\begin{array}{c}\text { Pods } \\
\text { per } \\
\text { cluster }\end{array}$ & $\begin{array}{c}\text { Pods } \\
\text { per } \\
\text { plant }\end{array}$ & $\begin{array}{c}\text { Seeds } \\
\text { per } \\
\text { pod }\end{array}$ & $\begin{array}{c}\text { Pod } \\
\text { length } \\
\text { (cm) }\end{array}$ & $\begin{array}{c}\text { Root- } \\
\text { shoot } \\
\text { length } \\
\text { ratio }\end{array}$ & $\begin{array}{c}\text { Root } \\
\text { Weight } \\
\text { per plant } \\
\text { (g) }\end{array}$ & $\begin{array}{c}\text { Seed } \\
\text { yield per } \\
\text { plant }(\mathrm{g})\end{array}$ & $\begin{array}{c}\text { Harvest } \\
\text { index }\end{array}$ \\
\hline 'UG157'/PU30' & 65.0 & 75 & 87 & 22.6 & 4.00 & 33 & 6.6 & 4.5 & 0.34 & 3.67 & 3.85 & 0.38 \\
\hline 'UG157'/'DPU915' & 35.3 & 80 & 89 & 27.0 & 5.00 & 66 & 7.0 & 5.1 & 0.38 & 2.00 & 6.60 & 0.45 \\
\hline 'UG157'/KAU7' & 55.0 & 73 & 83 & 12.6 & 4.00 & 28 & 6.6 & 4.6 & 0.37 & 2.51 & 3.63 & 0.39 \\
\hline 'PLU277'/'PU30' & 51.0 & 74 & 84 & 20.0 & 3.66 & 40 & 7.0 & 5.2 & 0.39 & 2.23 & 4.03 & 0.41 \\
\hline 'PLU277'/'DPU915' & 43.0 & 70 & 84 & 18.6 & 4.00 & 40 & 7.3 & 5.2 & 0.35 & 2.23 & 4.53 & 0.44 \\
\hline PLU277/'KAU7' & 31.0 & 71 & 84 & 13.3 & 4.33 & 35 & 6.3 & 4.5 & 0.35 & 1.66 & 5.45 & 0.49 \\
\hline 'PDB88-31'/PU30' & 41.6 & 73 & 85 & 17.6 & 3.66 & 34 & 6.3 & 4.7 & 0.28 & 1.66 & 5.52 & 0.51 \\
\hline 'PDB88-31'/'DPU915' & 37.0 & 79 & 91 & 24.3 & 4.00 & 51 & 6.6 & 4.0 & 0.33 & 1.66 & 9.00 & 0.53 \\
\hline 'PDB88-31'/KAU7' & 60.6 & 71 & 83 & 12.6 & 4.66 & 32 & 6.6 & 4.7 & 0.34 & 2.90 & 3.67 & 0.42 \\
\hline General Mean & 46.6 & 74 & 86 & 18.7 & 4.15 & 40 & 6.6 & 4.7 & 0.35 & 2.28 & 5.14 & 0.45 \\
\hline
\end{tabular}


Tab. 4. Estimates of $G C A$ effects of lines and testers in black gram

\begin{tabular}{|c|c|c|c|c|c|c|c|c|c|c|c|c|}
\hline & $\begin{array}{l}\text { Plant } \\
\text { height } \\
(\mathrm{cm})\end{array}$ & $\begin{array}{c}\text { Days } \\
\text { to } 50 \% \\
\text { flowering }\end{array}$ & $\begin{array}{l}\text { Days to } \\
\text { maturity }\end{array}$ & $\begin{array}{c}\text { Clusters } \\
\text { per } \\
\text { plant }\end{array}$ & $\begin{array}{c}\text { Pods } \\
\text { per } \\
\text { cluster }\end{array}$ & $\begin{array}{l}\text { Pods } \\
\text { per } \\
\text { plant }\end{array}$ & $\begin{array}{c}\text { Seeds } \\
\text { per } \\
\text { pod }\end{array}$ & $\begin{array}{l}\text { Pod } \\
\text { length } \\
(\mathrm{cm})\end{array}$ & $\begin{array}{l}\text { Root- } \\
\text { shoot } \\
\text { length } \\
\text { ratio }\end{array}$ & $\begin{array}{c}\text { Root } \\
\text { Weight } \\
\text { per } \\
\text { plant }(\mathrm{g})\end{array}$ & $\begin{array}{c}\text { Seed } \\
\text { yield per } \\
\text { plant }(\mathrm{g})\end{array}$ & $\begin{array}{c}\text { Harvest } \\
\text { index }\end{array}$ \\
\hline \multicolumn{13}{|c|}{ Lines } \\
\hline ‘UG157’ & $5.14^{*}$ & $2.04^{* *}$ & $0.89^{* *}$ & $1.92^{* *}$ & 0.19 & 2.44 & 0.04 & -0.01 & 0.01 & $0.44^{* *}$ & -0.45 & $-0.04^{* *}$ \\
\hline 'PLU277’ & $-4.96^{*}$ & $-2.29^{* *}$ & $-1.44^{* *}$ & $-1.41^{* *}$ & -0.14 & -1.56 & 0.14 & $-0.22^{*}$ & 0.01 & -0.24 & -0.47 & -0.01 \\
\hline 'PDB88-31' & -0.19 & 0.26 & $0.56^{* *}$ & -0.52 & -0.03 & -0.89 & -0.19 & $-0.23^{*}$ & $-0.04^{*}$ & -0.21 & $-0.92^{*}$ & $0.07^{* *}$ \\
\hline \multicolumn{13}{|c|}{ Testers } \\
\hline 'PU30’ & $5.92^{*}$ & -0.07 & -0.22 & $1.37^{*}$ & -0.36 & -4.33 & -0.08 & 0.07 & -0.01 & 0.24 & -0.68 & $-0.02^{* *}$ \\
\hline 'DPU915' & $-8.19^{* *}$ & $-2.15^{* *}$ & $-2.45^{* *}$ & $4.59^{* *}$ & 0.19 & $12.56^{* *}$ & 0.26 & 0.05 & 0.01 & $-0.32^{*}$ & $1.57^{* *}$ & $0.05^{* *}$ \\
\hline 'KAU7’' & 2.26 & $-2.07^{* *}$ & $-2.22^{* *}$ & $-5.97^{* *}$ & 0.19 & $-8.22^{* *}$ & -0.19 & -0.13 & 0.00 & 0.08 & $-0.89^{*}$ & -0.01 \\
\hline TS E (for lines) & 2.82 & 0.23 & 0.15 & 0.76 & 0.27 & 2.76 & 0.27 & 0.13 & 0.02 & 0.16 & 0.44 & 0.01 \\
\hline SE (for testers) & 2.82 & 0.23 & 0.15 & 0.76 & 0.27 & 2.76 & 0.27 & 0.13 & 0.02 & 0.16 & 0.44 & 0.01 \\
\hline
\end{tabular}

${ }^{*}{ }^{* *}$ Significant at $5 \%$ and $1 \%$ levels, respectively

On the other hand, the $S C A$ effect is a good estimate of nonadditive gene action (Rojas and Sprague, 1952). The cross 'PDB88-31'/'DPU915' was the best cross on the basis of per se performance in general. Positive significant $S C A$ effects of this cross for seed yield/plant and harvest index could be due to the parent 'DPU915' with good $G C A$ effect for four characters.

This indicated that additive gene action was predominant in the genetic control of seed yield/plant in this cross (Das and Das Gupta, 1999). The cross PLU277/'KAU7' appeared as the second best cross for seed yield/plant and harvest index showing positive significant $S C A$ effect and high per se performance. But both the parents of this cross were poor general combiners. It indicated that nonadditive gene action was predominant for these two traits in this cross. Both the top ranking crosses hold potential and could produce desirable transgressive segregants for seed yield and other quantitative traits in the subsequent segregating generations.

\section{Genetic parameters}

The magnitude of GCA and SCA variance (Tab. 6) for different quantitative traits revealed that both additive and nonadditive gene actions were important in the inheritance of seed yield and other quantitative traits in black gram. Similar results were reported by Singh and Singh (1971), Das Gupta and Das (1987), Chakraborty and Borua (1998), Dana and Das Gupta (2001). The relative magnitude of two variances computed by predictability ratio as suggested by Baker (1978) revealed the predominant role of nonadditive gene action for all the traits except clusters/plant.

The degree of dominance effect was higher than unity (1.00) except for clusters/plant (0.95). The estimate of narrow sense heritability was low for all the traits and ranged from 0.83 to $11.89 \%$. The estimate of predictability ratio for different quantitative traits revealed the predominance of nonadditive gene action except for clusters/plant. The depletion of additive gene action for the quantitative traits

Tab. 5. Estimates of $S C A$ effects of 9 crosses for various quantitative traits in black gram crosses

\begin{tabular}{|c|c|c|c|c|c|c|c|c|c|c|c|c|}
\hline Cross & $\begin{array}{l}\text { Plant } \\
\text { height } \\
(\mathrm{cm})\end{array}$ & $\begin{array}{c}\text { Days } \\
\text { to } 50 \% \\
\text { flowering }\end{array}$ & $\begin{array}{l}\text { Days to } \\
\text { maturity }\end{array}$ & $\begin{array}{l}\text { Clusters } \\
\text { per plant }\end{array}$ & $\begin{array}{l}\text { Pods } \\
\text { per } \\
\text { cluster }\end{array}$ & $\begin{array}{c}\text { Pods per } \\
\text { plant }\end{array}$ & $\begin{array}{c}\text { Seeds } \\
\text { per pod }\end{array}$ & $\begin{array}{c}\text { Pod } \\
\text { length }\end{array}$ & $\begin{array}{c}\text { Root- } \\
\text { shoot } \\
\text { length } \\
\text { ratio }\end{array}$ & $\begin{array}{c}\text { Root } \\
\text { Weight } \\
\text { per plant }\end{array}$ & $\begin{array}{c}\text { Seed } \\
\text { yield per } \\
\text { plant }\end{array}$ & $\begin{array}{c}\text { Harvest } \\
\text { index }\end{array}$ \\
\hline 'UG157’/PU30’ & 7.31 & $-0.93^{*}$ & $1.16^{*}$ & 0.57 & 0.03 & -5.25 & -0.10 & -0.27 & -0.01 & $0.70^{* *}$ & -0.16 & -0.01 \\
\hline 'UG157’‘DPU915’ & -8.28 & $1.45^{* *}$ & 0.19 & 1.75 & 0.48 & $11.16^{*}$ & -0.04 & 0.28 & 0.01 & -0.40 & 0.34 & -0.01 \\
\hline 'UG157’'KAU7’ & 0.98 & -0.60 & $-1.44^{* *}$ & $-2.39^{*}$ & -0.52 & -6.06 & 0.01 & 0.01 & 0.01 & -0.29 & -0.17 & -0.01 \\
\hline 'PLU277’'PU30’ & 3.42 & $2.40^{* *}$ & -0.11 & 1.30 & 0.02 & 6.05 & 0.20 & 0.13 & 0.04 & -0.05 & 0.04 & -0.01 \\
\hline 'PLU277’/'DPU915’ & $9.53^{*}$ & $-3.82^{* *}$ & $-2.48^{* *}$ & $-3.32^{*}$ & -0.19 & $-10.84^{*}$ & 0.16 & 0.22 & -0.02 & $0.51^{*}$ & $-1.71^{*}$ & $-0.05^{* *}$ \\
\hline ‘PLU277’/KAU7’ & $-12.91^{* *}$ & $1.40^{* *}$ & $2.49^{* *}$ & 1.94 & 0.14 & 4.64 & -0.39 & -0.33 & -0.01 & -0.46 & $1.67^{*}$ & $0.06^{* *}$ \\
\hline 'PDB88-31'/'PU30’ & $-10.76^{*}$ & $-1.49^{* *}$ & $-1.11^{* *}$ & -1.99 & -0.09 & -0.92 & -0.17 & 0.15 & -0.02 & $-0.65^{*}$ & 0.14 & 0.01 \\
\hline 'PDB88-31'/'DPU915’ & -1.25 & $2.29^{*}$ & $2.22^{* *}$ & 1.49 & -0.30 & -0.51 & -0.21 & $-0.50^{*}$ & 0.01 & -0.09 & $1.37^{*}$ & $0.04^{*}$ \\
\hline ‘PDB88-31'/KAU7’ & $11.91^{*}$ & $0.82^{*}$ & $-1.11^{* *}$ & 0.35 & 0.36 & 1.27 & 0.24 & 0.35 & 0.03 & $0.75^{* *}$ & $-1.50^{*}$ & $-0.09^{* *}$ \\
\hline $\mathrm{SE}(S C A$ effect $)$ & 4.89 & 0.39 & 0.26 & 1.31 & 0.48 & 4.77 & 0.47 & 0.23 & 0.04 & 0.28 & 0.77 & 0.02 \\
\hline
\end{tabular}


Tab. 6. Estimates of $G C A$ and $S C A$ variances and genetic parameters in black gram crosses

\begin{tabular}{|c|c|c|c|c|c|c|c|c|c|c|c|c|}
\hline Cross & $\begin{array}{l}\text { Plant } \\
\text { height } \\
(\mathrm{cm})\end{array}$ & $\begin{array}{c}\text { Days } \\
\text { to } 50 \% \\
\text { flowering }\end{array}$ & $\begin{array}{l}\text { Days } \\
\text { to } \\
\text { matu- } \\
\text { rity }\end{array}$ & $\begin{array}{c}\text { Clusters } \\
\text { per plant }\end{array}$ & $\begin{array}{l}\text { Pods } \\
\text { per } \\
\text { cluster }\end{array}$ & $\begin{array}{l}\text { Pods } \\
\text { per } \\
\text { plant }\end{array}$ & $\begin{array}{c}\text { Seeds } \\
\text { per } \\
\text { pod }\end{array}$ & $\begin{array}{l}\text { Pod } \\
\text { length }\end{array}$ & $\begin{array}{c}\text { Root- } \\
\text { shoot } \\
\text { length } \\
\text { ratio }\end{array}$ & $\begin{array}{c}\text { Root } \\
\text { Weight } \\
\text { per plant }\end{array}$ & $\begin{array}{c}\text { Seed } \\
\text { yield per } \\
\text { plant }\end{array}$ & $\begin{array}{c}\text { Harvest } \\
\text { index }\end{array}$ \\
\hline Cov H.S.(lines) & 28.66 & 1.91 & 0.37 & 0.36 & 0.26 & 26.01 & 0.00 & 0.006 & 0.0008 & 0.03 & 0.20 & 0.001 \\
\hline Cov H.S.(lines) & 0.64 & 1.63 & 3.51 & 26.64 & 0.19 & 91.41 & 0.02 & 0.047 & 0.0002 & 0.10 & 1.02 & 0.001 \\
\hline $6^{2} G C A$ & 3.66 & 0.44 & 0.39 & 3.38 & 0.06 & 8.17 & 0.003 & 0.006 & 0.0001 & 0.02 & 0.10 & 0.0002 \\
\hline $6^{2} \mathrm{~A}$ with $\mathrm{F}=1$ & 7.32 & 0.88 & 0.78 & 6.76 & 0.12 & 16.34 & 0.006 & 0.012 & 0.0002 & 0.04 & 0.20 & 0.0004 \\
\hline $6^{2} S C A$ (i.e. $\left.6^{2} \mathrm{D}\right)$ & 138.81 & 8.34 & 5.82 & 6.11 & 0.64 & 68.99 & 0.140 & 0.120 & 0.0010 & 0.46 & 1.90 & 0.0030 \\
\hline Predictability ratio & 0.05 & 0.11 & 0.13 & 1.11 & 0.19 & 0.24 & 0.04 & 0.10 & 0.20 & 0.09 & 0.11 & 0.13 \\
\hline Degree of dominance & 4.35 & 3.08 & 2.73 & 0.95 & 2.31 & 2.05 & 4.83 & 3.16 & 2.24 & 3.39 & 3.08 & 2.74 \\
\hline Type of dominance & OD & OD & OD & PD & OD & OD & OD & OD & OD & OD & OD & OD \\
\hline Narrow sense heritability (\%) & 3.42 & 7.93 & 11.78 & 11.89 & 10.71 & 5.86 & 0.83 & 3.15 & 1.18 & 7.84 & 4.03 & 2.86 \\
\hline
\end{tabular}

OD and PD denote overdominance and partial dominance, respectively

in black gram parents might be attributed to human selection for high yield (and consequently for other traits) over the past few centuries. Degree of dominance estimate resource-poor farmers of India for increasing black gram productivity. The use of genetic male sterility system will be effective in hybrid breeding program of black gram.

Tab. 7. Per cent contributions of lines, testers and their interactions to total variance of various traits in the black gram crosses

\begin{tabular}{ccccccccccccc}
\hline & $\begin{array}{c}\text { Plant } \\
\text { height } \\
(\mathrm{cm})\end{array}$ & $\begin{array}{c}\text { Days } \\
\text { to 50\% } \\
\text { flowering }\end{array}$ & $\begin{array}{c}\text { Days to } \\
\text { maturity }\end{array}$ & $\begin{array}{c}\text { Clusters } \\
\text { per } \\
\text { plant }\end{array}$ & $\begin{array}{c}\text { Pods } \\
\text { per } \\
\text { cluster }\end{array}$ & $\begin{array}{c}\text { Pods } \\
\text { per } \\
\text { plant }\end{array}$ & $\begin{array}{c}\text { Seeds } \\
\text { per } \\
\text { pod }\end{array}$ & $\begin{array}{c}\text { Pod } \\
\text { length }\end{array}$ & $\begin{array}{c}\text { Root-shoot } \\
\text { length ratio }\end{array}$ & $\begin{array}{c}\text { Root } \\
\text { Weight } \\
\text { per plant }\end{array}$ & $\begin{array}{c}\text { Seed } \\
\text { yield per } \\
\text { plant }\end{array}$ & $\begin{array}{c}\text { Harvest } \\
\text { index }\end{array}$ \\
\hline Lines (L) & 13.64 & 31.92 & 14.48 & 7.95 & 10.97 & 2.45 & 20.64 & 28.39 & 60.00 & 25.06 & 15.22 & 41.66 \\
Tester (T) & 28.56 & 29.99 & 49.84 & 78.09 & 39.02 & 64.96 & 38.09 & 6.65 & 16.67 & 13.69 & 44.64 & 16.68 \\
LX T & 57.80 & 38.09 & 35.68 & 13.96 & 50.01 & 32.59 & 41.27 & 64.96 & 23.33 & 61.25 & 40.14 & 41.66 \\
\hline
\end{tabular}

higher than unity for all the traits except clusters/plant indicated the role of overdominance in their expression. But the degree of dominance value of 0.95 indicated partial dominance for clusters/plant.

Narrow sense heritability is the proportion of additive genetic variance to total phenotypic variance. It reflects the fixable component of variance through selection leading to increased magnitude of a quantitative trait. Very low estimate of narrow sense heritability for all the traits indicated further, the predominance of nonadditive genetic variance in their expression. The per cent contributions of lines, testers and their interactions to the total variance of various quantitative traits in the black gram crosses were estimated (Tab. 7).

\section{Conclusions}

The results of the present study suggest that breeding methods such as recurrent selection or biparental mating followed by selection would be ideal to exploit both nonadditive and additive gene action for the quantitative traits in black gram. Since nonadditive gene action was predominant for most of the quantitative traits under the present study, heterosis breeding could also be used to harness it by producing and marketing hybrid varieties affordable to the

\section{Acknowledgements}

The authors are thankful to Assam Agricultural University, Jorhat, Assam, India for providing the fund and the facilities to undertake the present study at RARS, Nagaon.

\section{References}

Baker, R. J. (1978). Issues in diallel analysis. Crop Science 18:533-536.

Chakraborty, S. and P. K. Borua (1998). Inheritance of seed yield and its components in black gram (Vigna mungo L. Hepper). Indian Journal of Genetics and Plant Breeding 58:225-227.

Cheralu, C. A., N. Satyanarayana, K. Kulkarni, M. Jagdishwar, S. S. Reddy (1999). Combining ability analysis for resistance to preharvest sprouting in mungbean (Vigna radiata $\mathrm{L}$. Wilczek). Indian Journal of Genetics and Plant Breeding 59(4):465-472.

Dana, I. and T. Das Gupta (2001). Combining ability in black gram. Indian Journal of Genetics and Plant Breeding 61(2):170-171.

Das Gupta, T. and P. K. Das (1987). Genetics of yield in black 
126 gram. Indian Journal of Genetics and Plant Breeding 47:265-270.

Das, S. and T. Das Gupta (1999). Combining ability in sesame. Indian Journal of Genetics and Plant Breeding 59(1):69-75.

Haytowitz, D. B. and R. H. Mathews (1986). Composition of Foods: Legumes and Legume Products. Agricultural Handbook, USDA, USA.

Kempthorne, O. (1957). An Introduction to Genetical Statistics. John Wiley and Sons, Inc., New York.

Lauren, J. G., J. M. Duxbery, V. Beri, M. A. Razzaque II, M. A. Sattar, S. P. Pandey, S. Bhattarai, R. A. Mann and J. K. Ladha (1998). Direct and residual effects from forage and green manure legumes in rice-based cropping systems. In: J.V.D.K.Kumar Rao, C. Johanson and T. J. Rego (Eds.). Residual Effects of Legumes in Rice and Wheat Cropping Systems of the Indo-Gangetic Plain, Oxford and IBH, New Delhi.
Rojas, B. A. and G. F. Sprague (1952). A comparison of variance components in corn yield traits, III. General and specific combining ability and their interactions with locations and years. Agronomy Journal 44:462-466.

Singh, K. B. and J. K. Singh (1971). Heterosis and combining ability in black gram. Indian Journal of Genetics and Plant Breeding 31:491-498.

Sprague, G. F. and L. A. Tatum (1942). General versus specific combining ability in single crosses of corn. Journal of American Society of Agronomy 34:923-932. 